\title{
Animales y humanos en Las Cumbres de Ancasti (Siglos VIII y IX DC). Paisajes campesinos y recursos locales
}

\author{
ENRIQUE MORENO ${ }^{1-2} \&$ MAXIMILIANO AHUMADA $^{1}$ \\ ${ }^{1}$ CITCA (CONICET-UNCA) \\ ${ }^{2}$ Escuela de Arqueología, UNCA. \\ enalmor@gmail.com; maximilianoahumada@hotmail.com
}

(Recibido 20 septiembre 2017; Revisado 5 febrero 2018; Aceptado 8 marzo 2018)

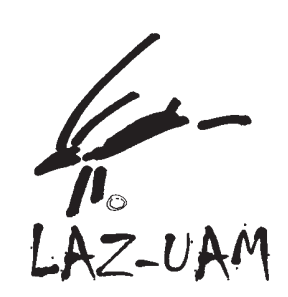

RESUMEN: La sierra de El Alto-Ancasti en el oriente de la provincia de Catamarca (Argentina) presenta una importante variabilidad paisajística que se traduce en una variada presencia de recursos faunísticos. Aunque el paradigma imperante definió a las poblaciones indígenas como pastores de camélidos, recientes investigaciones han evidenciado ocupaciones campesinas de larga duración y con un fuerte compromiso con el entorno local y sus recursos. En este trabajo presentaremos la información obtenida en el análisis faunístico del sitio El Taco 19 (ET19), entre los siglos VIII y IX d.C., que posee una representación importante de camélidos domesticados, pero también un rico registro de especies silvestres, implicando diferentes estrategias de aprovechamiento de la fauna. El estudio permitió reconstruir una historia donde los animales jugaron un rol importante como recursos económicos y estructuradores de las relaciones sociales. De esta suerte, como valoraremos en este trabajo, el pastoreo y la caza, constituyen prácticas centrales para comprender los vínculos establecidos entre animales y personas en las serranías del este catamarqueño.

PALABRAS CLAVE: ANIMALES, PASTOREO, CAZA, CAMÉLIDOS, PAISAJE CAMPESINO

ABSTRACT: The El Alto-Ancasti mountains in the eastern sector of the province of Cajamarca (Argentina), features a varied landscape along with a varied presence of faunal resources. The traditional view has been to consider these populations as camelids herders, though recent researches evidenced long-term rural occupations with a strong commitment with the local environment and its resources. This work discusses the results from a faunal analysis carried out on the VII-IX AD occupations at El Taco 19 (ET19). This sites features an abundance of domesticated camelids along with wild species, thus implying different exploitation strategies. This study allowed us to reconstruct a history where animals played important roles, both as economic resources and crucial elements of the social relationships. Herding and hunting thus emerge as central practices to understand the links established between animals and people present in the highlands of El Alto-Ancasti.

KEYWORDS: ANIMALS, HERDING, HUNTING, CAMELIDS, PEASANT LANDSCAPE 


\section{INTRODUCCIÓN}

Considerando las características ambientales y ecológicas de la cumbre de la Sierra de El Alto-Ancasti, resulta lógico imaginarlas como un espacio óptimo para el establecimiento de prácticas pastoriles. Se trata de un paisaje dominado por un piso ecológico de pastizal de altura, surcado por quebradas, en las que se puede encontrar, en las zonas más bajas, cursos de agua, algunos temporales y otros permanentes. Incluso hoy, en el área se puede observar una gran presencia de vacunos y equinos a lo largo de este territorio. Probablemente esta situación haya sido una de las principales causas que motivó la visión de este paisaje como un espacio casi exclusivamente ganadero a través del tiempo, que fuera trasladado hacia el pasado, para entender la ocupación humana de esta área. Esta situación se vinculó especialmente al Período de Integración Regional durante el cual dicha región fue pensada como un área importante considerando los variados espacios rupestres, la presencia de bosques de cebil, articulándose a cabeceras políticas-administrativas ubicadas en valles aledaños (Pérez Gollán, 1994; Kriscautzky, 1996-97; Nazar, 2003). De esta manera, sería un espacio con una arquitectura vinculada exclusivamente a la producción pastoril, y con muy baja densidad poblacional, la cual se dedicaría únicamente al cuidado de la hacienda y su transporte, así como también con cierta estacionalidad vinculada a dicha producción. Esta visión de la ocupación prehispánica de la Sierra, ha sido discutida por el avance de distintas investigaciones en la zona (Nazar, 2003; Dlugosz, 2005; Gordillo et al., 2010; Quesada, 2011; Taboada, 2011; Meléndez \& Quesada, 2012; Moreno \& Quesada, 2012; Moreno \& Sentinelli, 2012; Quesada et al., 2012; Zuccare1li, 2012; Gordillo \& Zuccarelli, 2013; Granizo \& Barot, 2014; Moreno, 2014; Ahumada y Moreno, 2015-2016; Moreno \& Egea, 2016), mostrando una ocupación campesina de larga duración, con presencia de una arquitectura doméstica compleja y una transformación del paisaje para la preparación de suelos agrícolas. Sin embargo, esto no implica la inexistencia del pastoreo como una actividad relevante para estas poblaciones, sino que el foco debe ser puesto en su rol dentro de una lógica de producción y reproducción campesina y no ya considerándola como una periferia productora de recursos para centros políticos-administrativos. Ahora bien, entendiendo la ocupación humana de esta región desde una visión que no se sustenta en supuestos ecológico-políticos, sino más bien en la interacción entre poblaciones humanas, entorno, recursos, seres no humanos, etc. y en la conformación de mundos de la vida a partir de la rutinización y construcción de relaciones cotidianas, es que pretendemos entender aquí de qué manera se vincularon a través de dos prácticas particulares, la caza y el pastoreo, las poblaciones humanas y los animales que habitaron este paisaje. Para ello, debemos partir de la información obtenida de los conjuntos faunísticos recuperados del área de estudio y vincularlo con las interpretaciones hasta hoy alcanzadas acerca de la vida cotidiana en dicho lugar. Pero antes de desandar este camino, creemos que es necesario preguntarnos en términos generales ¿cómo podemos pensar las relaciones entre humanos y animales?

\section{RELACIONANDO ANIMALES Y HUMANOS}

Son múltiples las formas en que han sido pensadas las relaciones entre humanos y animales a través del tiempo. Podemos decir que existe una visión prevaleciente, que tiende a pensar a los animales como una fuente de recursos diversos, sobre todo proteicos, vinculados al ámbito económico de reproducción social. De esta manera, se prioriza en la relación el beneficio alimenticio o de recursos secundarios que las poblaciones humanas pueden obtener de la explotación animal. Son varios los ejemplos de cálculos de rendimiento, vínculos entre el costo de obtención del recurso y el rédito que se obtenía del mismo, buscando definir estrategias para optimizar la toma de decisiones (por ejemplo, Binford, 1980; Bettinger, 1991). En esta visión prevalece el aspecto económico de la relación. Sin embargo, se han propuesto otras formas de relacionarse entre animales y humanos. Desde diferentes ópticas teóricas y metodológicas se ha planteado pensarla entre seres con agencias particulares donde sus relaciones pueden ser comprendidas en estos términos y no suponiendo la preminencia del humano frente al resto de la naturaleza. Esta perspectivas tienden a modificar el foco de la explicación en torno de la importancia de la reproducción humana frente a los recursos naturales, pretendiendo pensar un fenómeno particular, como puede ser el cazar un animal, en el marco de una red de relaciones entre seres actuantes, donde los saberes y conocimientos mutuos juegan un rol preponderante (Ingold, 1987, 
1988; Viveiros de Castro, 1996, 2004; Marciniak, 1999; Arhem, 2001; Descola, 2004).

También, se han planteado múltiples visiones acerca de la íntima relación que se plasma entre los animales domésticos y sus dueños, en donde se dan relaciones de amor y cariño entre ellos que dan sentido a la relación (Arnold \& Yapita, 1998; Haber, 2006, 2007; Tomasi, 2011; Bugallo \& Tomasi, 2012).

Sin embargo, no pretendemos aquí hacer una detallada presentación y discusión de estas posturas, sino más bien llamar la atención sobre la diversidad de miradas sobre el asunto.

Nuestra posición parte de la idea que las relaciones entre humanos y animales debe ser entendida en términos múltiples, entendiéndola no sólo como un factor económico que permite la reproducción social, sino que en ella juegan un rol imprescindible decisiones y elecciones que tienen que ver con aspectos sociales, políticos, religiosos, etc. Pero además, entendemos que las formas de apropiación de las distintas especies de animales implican una primera diferencia clara. La cacería y el pastoreo son dos formas muy diferentes de relacionar humanos y animales, pero también de comprender la relación entre las personas, ya que ambas implican divergencias en torno a la toma de decisiones vinculadas al sacrificio de un animal y a la utilización de sus recursos (Ingold, 1987, 1988; Vicent García, 1991). Estamos de acuerdo en que siempre el objetivo final tanto del pastoreo como de la cacería es el obtener recursos para la subsistencia y, por lo tanto, la reproducción social, pero las formas en que se dan estas prácticas, quiénes participan, cómo se accede al recurso, que animales se cazan, etc. responde a una multiplicidad de factores sociales que es imprescindible considerar.

\section{PASTOREO Y CAZA EN LA CUMBRE DE EL ALTO-ANCASTI}

Uno de los principales aspectos que resaltó al iniciar las investigaciones en la cumbre de la Sierra de El Alto-Ancasti (Figura 1) fue una multiplicidad de estructuras habitacionales y productivas, con una alta inversión de trabajo, que contrastaba con la visión de una ocupación temporaria dirigida a la producción ganadera para centros políticos-administrativos. Frente a esto, sitios como El Taco 19 (ET19) presentaban 27 recintos de distintos tama- ños, algunos muy grandes, que podrían ser patios o espacios a cielo abierto para la realización de diversas actividades y otros más pequeños, interpretados como habitaciones (Figura 2). Esta arquitectura

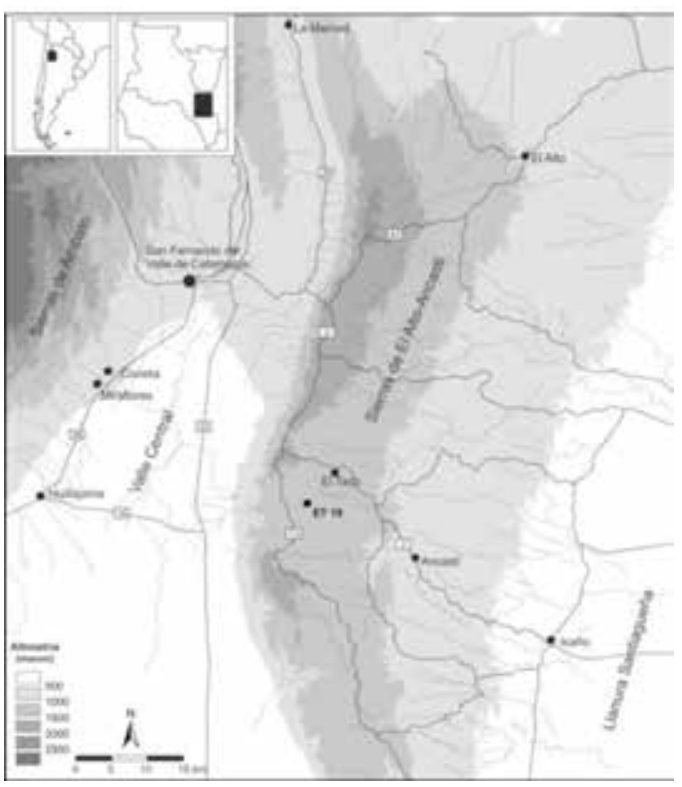

FIGURA 1

Mapa de localización de la Sierra de El Alto-Ancasti.

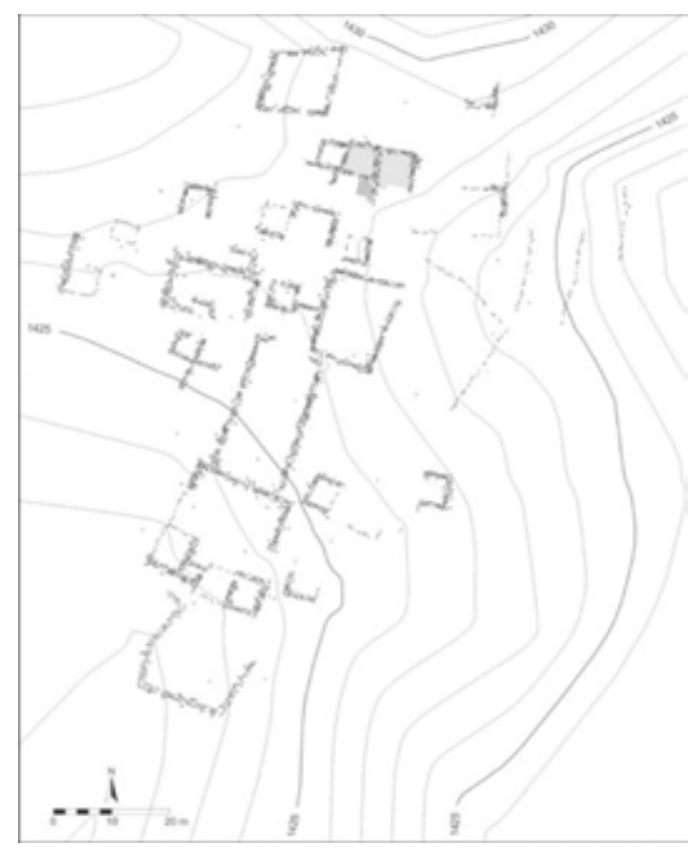

FIGURA 2

Plano del sitio ET19. En gris se indican los recintos excavados. 


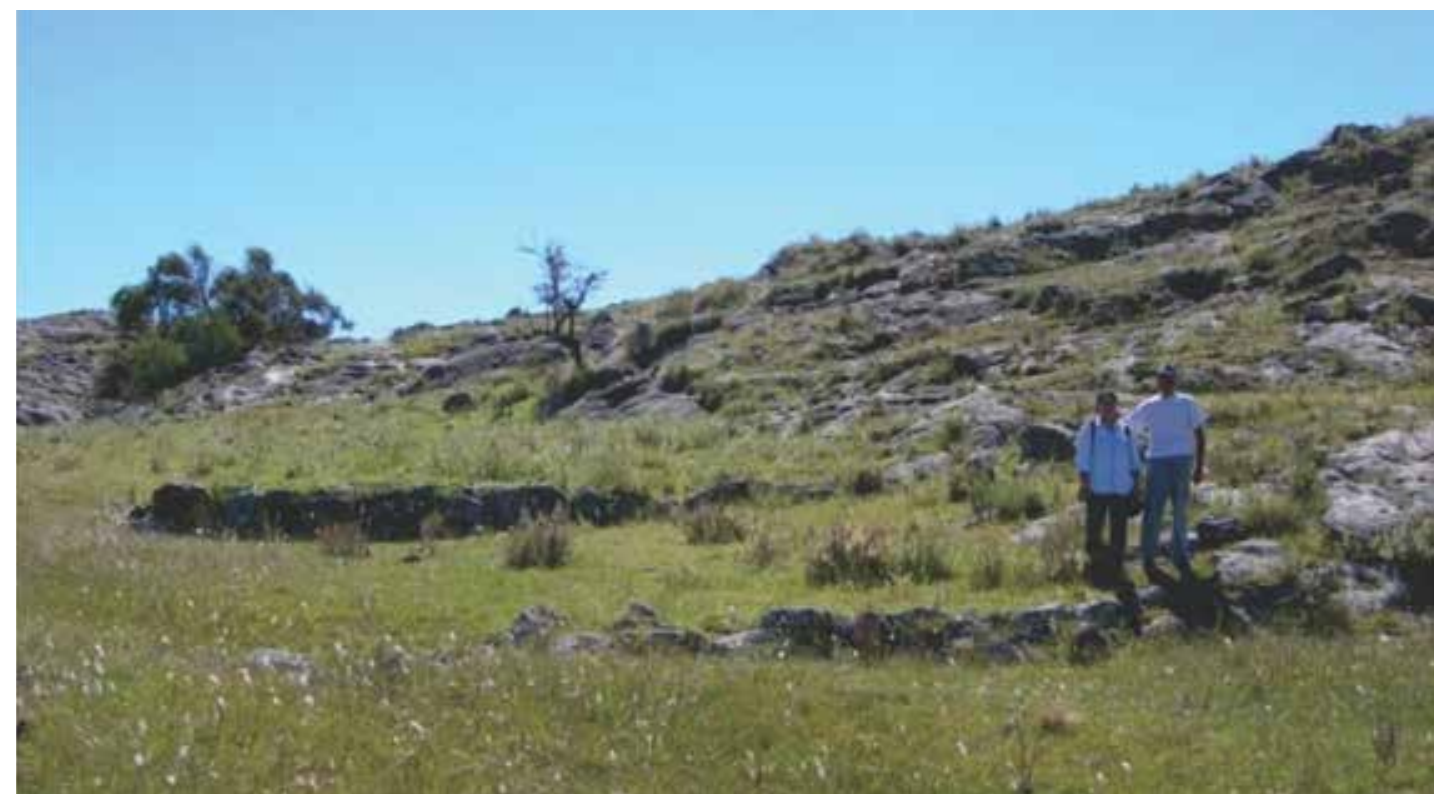

FIGURA 3

Ejemplo de terrazas de cultivo identificadas en la zona de El Taco.

doméstica, se articulaba con una fuerte presencia de construcciones agrícolas, especialmente terrazas de cultivo (Figura 3), que fueron identificadas, prácticamente, en todas las quebradas que descendían desde la cumbre hasta los sectores más bajos irrigados por cursos de agua temporales o permanentes (Quesada et al., 2012).

Asumiendo una ocupación permanente de estos espacios, teniendo en cuenta la inversión de trabajo en la construcción de cada uno de estos conjuntos, pero también la gran cantidad de conjuntos de características semejantes observados en un espacio de aproximadamente 540 Ha. (Figura 4) (Quesada et al., 2012), nos propusimos caracterizarlos a partir de la realización de una excavación sistemática de dos recintos del sitio ET19, con el objetivo de entender su dinámica de ocupación y otorgar profundidad histórica al mismo.

Los espacios excavados en ET19, fueron los recintos R1 y R2 y un espacio externo vinculado al ingreso a R1. La arquitectura de este conjunto presenta muros con una secuencia constructiva, cuya base presenta una doble hilera de lajas ubicadas de forma vertical clavadas en el sedimento, que puede alcanzar una altura de $1 \mathrm{~m}$, completadas por la colocación de bloques irregulares, aunque seleccionando las caras más regulares hacia el interior del recinto. El intersticio entre las lajas era completado con tierra, mientras que se observa mortero en el caso de los bloques que completan los muros (Figura 5).

El Recinto 1 presenta un tamaño aproximado de $5 \mathrm{~m}$ de lado con una abertura hacia el $\mathrm{S}$, mientras que el Recinto 2 presenta mayores dimensiones y hasta el momento no se ha podido observar la pared de cerramiento hacia el S, por lo que fue interpretado como un posible espacio abierto, probablemente sin techar o con techado parcial, similar a un patio o una galería. Además, se inició la excavación de un espacio exterior asociado a la abertura del Recinto 1. Hasta el momento contamos con cuatro fechados radiocarbónicos obtenidos en los Recintos 1 y 2 que ubican la ocupación humana de estos recintos en la segunda mitad del primer milenio de la era (Tabla 1), contrastable con la información estilística de la cerámica recuperada, así como también de la arquitectura del sitio. Particularmente en relación a la cerámica recuperada, se observan fragmentos asignables a estilos ubicados en dicho período histórico tales como Aguada Portezuelo, Ambato tricolor, Candelaria, Condorhuasi bicolor, Ambato negro grabado, Ciénaga, Aguada pintado (Barot, 2017).

El Recinto 1 de este conjunto ha sido interpretado como un área de ocupación habitacional donde pudieron identificarse diversos pisos de ocupación, así como también modificaciones estructurales, 


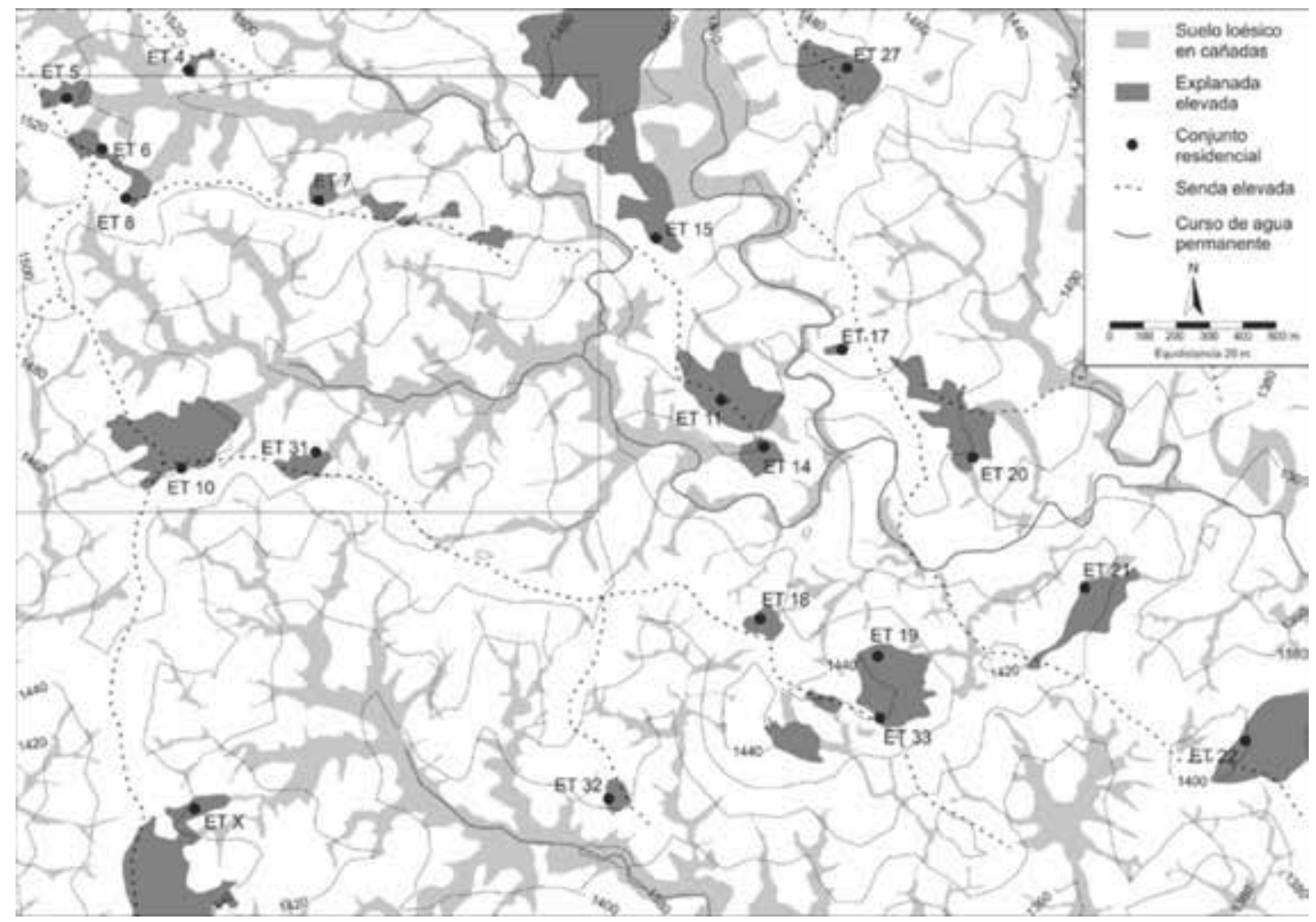

FIGURA 4

Mapa de distribución de los sitios identificados en la zona de El Taco (tomado de Quesada et al., 2012).

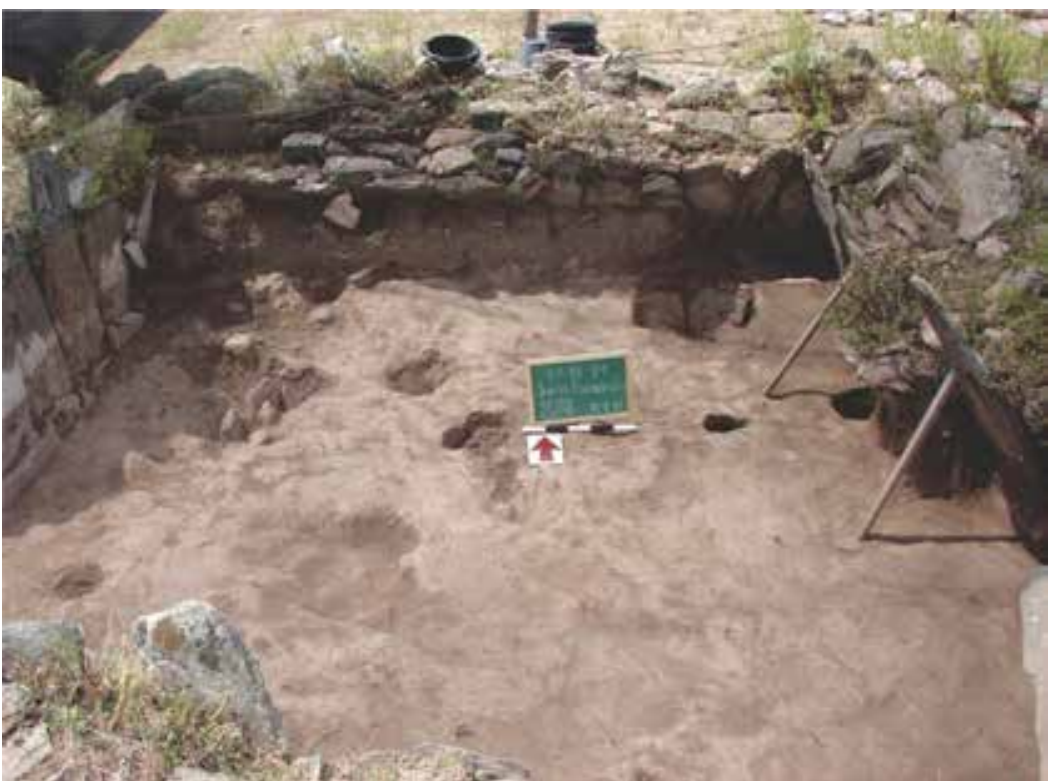

FIGURA 5

Vista general del Recinto 1 de ET19. Nótese la presencia de las lajas verticales clavadas, de las cuales algunas superan el metro de altura. 
como la clausura de un vano y la apertura de otro en otra posición. Por su parte el Recinto 2 que se encuentra abierto hacia el Sur, fue interpretado como un área de actividades y vertedero de desechos del Recinto 1. Incluso su construcción es posterior al Recinto 1, tal como lo muestra la unión de los muros en el sector noroeste del Recinto 2, así como también en la secuencia estratigráfica de ambos recintos (Quesada \& Gastaldi, 2013).

\begin{tabular}{|c|c|}
\hline $1390 \pm 70 \mathrm{AP}$ & (LATYR-2583; hueso) \\
\hline $1270 \pm 60 \mathrm{AP}$ & (LATYR-2924; hueso) \\
\hline $1240 \pm 50 \mathrm{AP}$ & (LATYR-2735; hueso) \\
\hline $1340 \pm 80 \mathrm{AP}$ & (LATYR-2930; hueso) \\
\hline
\end{tabular}

TABLA 1

Fechados radiocarbónicos obtenidos en ET19.

Durante la excavación se recuperaron numerosos materiales arqueológicos que han aportado información relevante acerca de las prácticas realizadas en el interior de estos recintos, así como también de la importancia de la vinculación con los recursos locales por parte de estas poblaciones (Moreno \& Quesada, 2012; Moreno \& Sentinelli, 2012; Moreno, 2014; Ahumada y Moreno, 20152016; Barot, 2017).

\section{EL CONJUNTO ÓSEO EN ET19}

En el caso particular de los materiales faunísticos, el número total de restos es de 854 , donde resalta cierta variabilidad de taxones representados, lo cual nos obliga a indagar en las diferentes maneras en que animales y personas se vincularon en este sitio.

Pero antes de introducirnos en este tópico, presentaremos algunos de los datos generales relevantes acerca del conjunto. En primer lugar, debemos mencionar que la muestra presenta un estado de conservación buena, en mayor medida el registro se encuentra entre los estadios uno y dos propuestos por Behrensmeyer (1978), más precisamente se trata del $53 \%$ de la muestra, un $39 \%$ en estadio tres y el resto corresponden al estadio cuatro. No observamos marcas de raíces y en cuanto a las marcas realizadas por roedores y carnívoros debemos decir que sólo el 3,16 \% ( $\mathrm{n}=21$ a roedores y n=6 a carnívoros) de la muestra ha sido afectada (Binford, 1981; Mengoni Goñalons, 1988, 1999;
Lyman, 1994; Reitz \& Wing, 2008). En cuanto a la presencia de huellas de corte o percusión, este valor es claramente más alto, alcanzando el 15\% del conjunto en general, así como las huellas de termoalteración alcanza un valor semejante (Mengoni Goñalons, 1988, 1999; Lyman, 1994; Chaix \& Meniel, 2005; Reitz \& Wing, 2008). Resumiendo, el conjunto ha sido afectado por agentes tafonómicos, coherente con las características ambientales del área de estudio, pero mantiene condiciones que permiten obtener información relevante.

Del conjunto total, logramos identificar un NISP de $516(60,4 \%)$. Como mencionamos antes, contamos con una representación de taxones que pueden implicar distintos relacionamientos con las poblaciones asentadas en ET19. Comenzando con los menos representados, observamos algunos especímenes de fauna exótica como un espécimen de costilla de Capra hircus, una primera falange de Bos taurus, ubicados en los estratos superiores de la excavación vinculados a momentos posteriores al derrumbe de ET19 y por lo tanto intrusivos de momentos contemporáneos ${ }^{1}$. En el resto del conjunto se observan taxones con menor representación como por ejemplo un molar de Pseudalopex sp., seguidos de cérvidos $(1,36 \%)$, aves $(2,71 \%)$, mamíferos grandes $(6,59 \%)$, roedores $(11,82 \%)$ y Camelidae $(76,89 \%)$ (Tabla 2).

\begin{tabular}{|c|c|c|}
\hline TAXÓN & $\mathrm{N}$ & $\%$ \\
\hline Camelidae & 397 & 76,94 \\
\hline Roedores & 61 & 11,82 \\
\hline Pseudalopex sp. & 1 & 0,19 \\
\hline Bos taurus & 1 & 0,19 \\
\hline Capra hircus & 1 & 0,19 \\
\hline Ave & 14 & 2,71 \\
\hline Mamifero & 7 & 1,36 \\
\hline & 34 & 6,59 \\
\hline Total & 516 & 100 \\
\hline
\end{tabular}

TABLA 2

Taxones presentes en ET19.

Lo que resalta de la identificación taxonómica del conjunto de ET19 es la alta presencia de Ca-

\footnotetext{
${ }^{1}$ Todos los especímenes óseos provenientes de las unidades estratigráficas identificadas como posteriores al derrumbe de ET19 fueron dejados de lado en el presente análisis.
} 
melidae, frente a otros taxones, y por ello comenzaremos comentando las características de este conjunto, para luego evaluar la presencia de los taxones menos representados y su articulación con la reproducción social en ET19.

Retomando el núcleo central de este trabajo, a continuación evaluaremos las diferentes prácticas a través de las cuales se habrían materializado las relaciones entre humanos y animales en cumbre de la Sierra de El Alto-Ancasti.

\section{PASTOREO}

En base a la información comentada antes, resulta claro que una de las formas prioritarias de vincularse entre humanos y animales en las cumbres de la sierra, fue a través de la crianza de la hacienda. Existen muchos estudios acerca de lo que implica la relación entre pastores y animales a lo largo de los Andes y su implicancia en torno de una red de significados sociales que dista de ser un mero hecho económico de obtención de recursos por parte de animales de su propiedad (Nielsen, 1996, 2009; Arnold \& Yapita, 1998; Haber, 2006, 2007; Tomasi, 2011; Bugallo \& Tomasi, 2012).

El pastoreo puede ser pensado como una práctica social vinculada con la crianza de los animales, pero que a su vez permite y sugiere la reproducción del grupo social. En este sentido, la noción de crianza, se hermana con la idea de criar a un hijo, con cariño, respeto, etc. en dónde no sólo el objetivo es obtener recursos, sino también se asienta en una relación entre sujetos activos que supera una mera visión económica (Haber, 2006, 2007). En este vínculo se insertan relaciones de cuidado y cariño y que además se encuentran mediadas por otros seres, como la Pachamama u otras deidades propiciatorias en una compleja red de relaciones imprescindibles para la reproducción social.

En este marco, es importante evaluar cuáles serían las evidencias presentes en la cumbre de El Alto-Ancasti para pensar en la relevancia del pastoreo como práctica social. El primer dato que resulta relevante es la presencia de restos óseos de camélidos, tal como comentamos antes, en los materiales recuperados de la excavación de ET19. Al avanzar en un análisis taxonómico de los camélidos presentes, observamos tres tendencias de tamaño de los restos óseos muy clara. Para ello sólo tuvimos en cuenta las medidas de las $1^{\circ}$ falanges. En este conjunto, uno de los especímenes representa un animal pequeño, casi seguramente vicuña, mientras que los otros dos grupos de tamaño se vinculan a medidas correspondientes a llama/guanaco pero donde dos piezas presentan cierta discordancia en el ancho latero-medial de la $1^{\circ}$ falange (Figura 6)

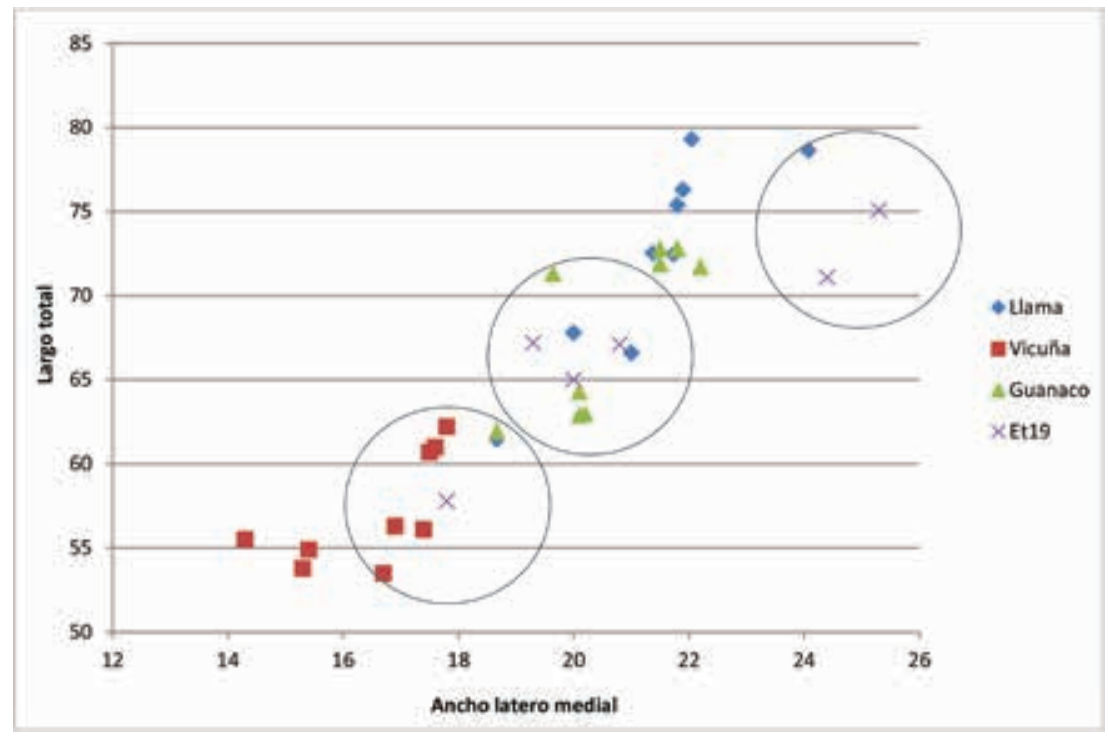

FIGURA 6

Comparación de la osteometría de falanges proximales (ancho latero-medial de la epífisis proximal y largo total, ambas medidas en mm) en ET19. Las medidas de referencia fueron tomadas de Izeta et al. (2009). 


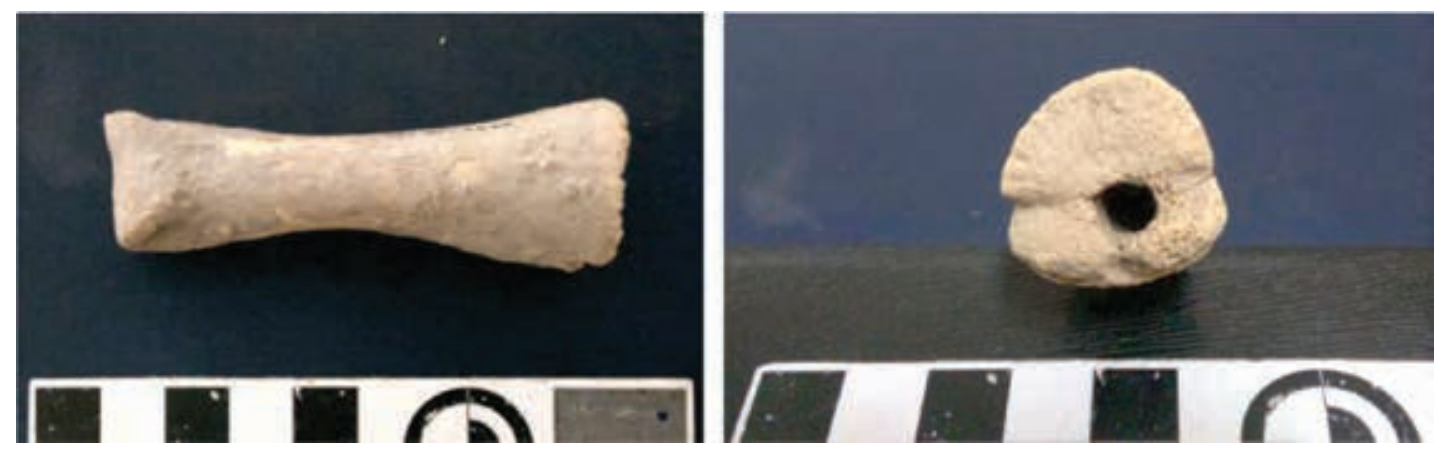

FIGURA 7

Ejemplar de $1^{\circ}$ falange correspondiente al grupo de mayores dimensiones y que se encuentra perforado de manera longitudinal.

en relación a los datos de referencia que utilizamos (Izeta et al., 2009). Además, estas dos falanges comparten una perforación en sentido longitudinal (Figura 7).

La identificación taxonómica no es determinante en cuanto a la diferenciación entre llamas y guanacos en la zona cumbral de la sierra. Sin embargo, la representación de partes esqueletarias es un aspecto que nos permite acercarnos un poco más a esta cuestión. En la Tabla 3 se presentan los valores del NISP, MNE, MNI y MAU\% de aquellos especímenes asignados a camélidos. En los mismos se observa una representación bastante equilibrada del esqueleto axial y del apendicular, notándose el ingreso tanto de aquellas partes con alto contenido de carne y bajo contenido de médula (vértebras y costillas), así como también de aquellas partes con mayores valores de médula y cantidades entre altas y bajas de carne (fémur, radio-cúbito, falanges, metapodios) (Mengoni Goñalons, 1991; Yacobaccio et al., 1997-1998). Al analizar estos valores en relación al MNI, se pude observar una mayor representación del esqueleto apendicular, particularmente de las patas traseras y delanteras, lo que indicaría cierto interés por la obtención de médula. Sin embargo, además identificamos varios restos de cráneo, mandíbula y carpianos y tarsianos, que serían partes de menor rinde económico. Esto creemos, disminuye las posibilidades de que sean elementos transportados y, por lo tanto, que el lugar de muerte de los animales haya sido cercano a ET19, pensando en que serían animales domésticos y que se aprovechara por ello todos los recursos obtenibles de los mismos (Figura 8). La representación de las partes esqueletarias de camélidos, indica la presencia de todo el esqueleto prácticamente, que difiere considerablemente de lo observado en los pocos especímenes identificados de animales sil- vestres, en donde predominan, pelvis, y huesos largos únicamente, sin haberse registrado cráneos, vértebras y costillas.

\begin{tabular}{|l|c|c|c|c|c|}
\hline Parte & NISP & MNE & MNI & MAU & MAU \% \\
\hline Cráneo & 20 & 2 & 2 & 2,00 & 66,67 \\
\hline Incisivo & 4 & 3 & 1 & 1,00 & 33,33 \\
\hline Molar & 5 & 2 & 1 & 0,67 & 22,22 \\
\hline Cervical & 8 & 4 & 1 & 0,80 & 26,67 \\
\hline Dorsal & 1 & 1 & 1 & 0,08 & 2,56 \\
\hline Lumbar & 4 & 3 & 1 & 0,50 & 16,67 \\
\hline Costilla & 47 & 22 & 2 & 0,85 & 28,21 \\
\hline Escápula & 4 & 3 & 2 & 1,50 & 50,00 \\
\hline Húmero & 8 & 4 & 2 & 2,00 & 66,67 \\
\hline Radio-Cúbito & 7 & 5 & 3 & 2,50 & 83,33 \\
\hline Magnum & 3 & 3 & 2 & 1,50 & 50,00 \\
\hline $1^{\circ}$ falange & 12 & 9 & 2 & 1,13 & 37,50 \\
\hline $2^{\circ}$ falange & 9 & 7 & 1 & 0,88 & 29,17 \\
\hline $3^{\circ}$ falange & 4 & 3 & 1 & 0,38 & 12,50 \\
\hline Pelvis & 2 & 2 & 2 & 2,00 & 66,67 \\
\hline Fémur & 8 & 6 & 3 & 3,00 & 100,00 \\
\hline Patela & 3 & 3 & 2 & 1,50 & 50,00 \\
\hline Tibia & 7 & 4 & 2 & 2,00 & 66,67 \\
\hline Navicular & 6 & 5 & 2 & 2,50 & 83,33 \\
\hline Astrágalo & 5 & 3 & 2 & 1,50 & 50,00 \\
\hline Metacarpo & 1 & 1 & 1 & 0,50 & 16,67 \\
\hline Metatarso & 2 & 2 & 1 & 1,00 & 33,33 \\
\hline Metapodio & 19 & 11 & 3 & 2,75 & 91,67 \\
\hline
\end{tabular}

TABLA 3

Medidas de cuantificación del conjunto óseo de ET19.

Otro aspecto importante sería el perfil etario identificado en el conjunto de camélidos (Wing, 1972; Mengoni Goñalons, 1988; Wheeler, 1999), en el cual podemos observar que 13 especímenes se corresponden con animales de menos de 12 meses de vida, mientras que un conjunto de 13 especímenes se ubican entre los 0 y 36 meses de vida y 
8 entre los 0 y 42 meses de vida. En relación a los especímenes maduros se observa que 30 especímenes presentan su epífisis fusionados, de los cuales 24 se corresponden con animales de más de 24 meses de vida (Tabla 4). Esto indicaría una estrategia que buscaría principalmente la obtención de carne y médula, aunque también la presencia de especímenes maduros se vincularía a la obtención de lana. Los valores obtenidos en la muestra de ET19 son semejantes a los presentados por Dantas (2010) para el valle de Ambato, aunque con la diferencia sustancial de que en el caso de Ambato, existe una baja representación de las partes de mayor índice de utilidad del esqueleto axial como ser vértebras y costillas, mientras que en ET19, estos especímenes se encuentran bastante representados.

\begin{tabular}{|c|c|c|c|}
\hline Espécimen & Maduro & Inmaduro & Meses \\
\hline Pelvis & 1 & 2 & $12-18$ \\
\hline Tibia y Húmero distal & 4 & 7 & $12-18$ \\
\hline Radio-Cúbito proximal & 1 & 4 & $12-18$ \\
\hline Metapodio distal & 3 & 7 & -32 \\
\hline Falanges & 18 & 6 & $20-36$ \\
\hline $\begin{array}{c}\text { Radio-Cúbito distal, } \\
\text { Fémur proximal, Tibia } \\
\text { proximal }\end{array}$ & 3 & 8 & -42 \\
\hline Total & 30 & 34 & \multicolumn{2}{|l}{} \\
\cline { 1 - 3 } & \multicolumn{2}{|l}{} &
\end{tabular}

TABLA 4

Perfil etario del conjunto de camélidos registrados en ET19.

Otro aspecto relevante acerca del pastoreo en esta zona, sería la presencia de amplios pastizales de gran distribución de excelente calidad y que puedan explicar un manejo de este tipo, asociado a las demás prácticas implementadas por estas poblaciones durante los siglos VIII y IX d.C. La cumbre de la sierra de El Alto-Ancasti, presenta una amplia cobertura, caracterizada principalmente por paja (Jarava sp.), pasto horqueta (Paspalum notatum) y gramilla (Cynodon dactylon), que presentan calidades muy alta para el manejo de hacienda (Emiliano Quiroga, INTA, comunicación personal, Agosto 2017) y que se distribuye por un amplio territorio de la zona cumbral de la sierra, tal como se puede observar en la Figura 8. Por lo tanto sería un espacio en el cual sería viable el manejo de camélidos domésticos. En este sentido, esta vinculación del paisaje con prácticas sociales a escala local, va de la mano de la información obtenida de los análisis del paisaje tanto doméstico como agrícola del área (Quesada et al., 2008, 2012), así como también del material lítico recuperado hasta el momento en ET19 (Moreno \& Sentinelli, 2012; Moreno, 2014).

Pero volviendo a lo comentado hacia el inicio de este trabajo y considerando la presencia de restos faunísticos correspondientes a llamas, la interpretación de los sitios ¿por qué no podría vincularse con aquella que asumía un rol exclusivamente pastoril a las ocupaciones humanas de la cumbre de El Alto-Ancasti? Creemos que esta situación no es así por varias razones. En primera instancia, por la arquitectura doméstica y productiva registrada en ET19 y en otros sitios del área. Estos espacios han mostrado una preparación de la estructuras habitacionales, con una clara intención de permanecer en el tiempo, vinculado a múltiples prácticas sociales, incluido el pastoreo, que implicó, por ejemplo, la selección, transporte, colocación de lajas en la base de los muros, que en algunos casos sobrepasa el metro de altura, y que suponen una fuerte inversión de trabajo, el acondicionamiento del espacio doméstico, que difícilmente pueda responder sólo a un lugar preparado para el cuidado de los rebaños y para la permanencia estacional de los pastores (Quesada et al., 2012). En la misma tónica se sitúan los múltiples espacios aterrazados, ubicados en prácticamente todas las quebradas que descienden desde las cumbres hacia los cauces hídricos. Estas estructuras, al igual que los conjuntos domésticos, implicaron la preparación del terreno para su construcción, el traslado de rocas y una inversión de trabajo en la construcción de los muros, así como también en la nivelación del espacio agrícola (Quesada \& Zuccarelli, 2016).

Otro aspecto tiene que ver con un supuesto ecológico acerca del desarrollo social en el Noroeste Argentino, el cual presupone y predispone a las Sierras de El Alto-Ancasti, por sus características ambientales y su caracterización histórica, como un espacio pastoril. Esta visión se encuentra sustentada en las características ocupacionales de los siglos XIX y XX d.C. donde los pastizales de altura fueron utilizados para la producción de ganadería que luego era trasladada al norte de Córdoba para su exportación. Esta construcción, que ya ha sido discutida en otros trabajos (Quesada, 2011; Quesada et al., 2012; Ahumada \& Moreno, 2015-2016), anularía la posibilidad de pensar en poblaciones locales viviendo y produciendo para ellas mismas en esta locación.

Por el contrario, en base a la información faunística comentada antes, la práctica pastoril en El Taco se habría vinculado más bien con la presen- 
cia de rebaños controlados por los pobladores de ET19, como parte de una práctica cotidiana más sumada a otras como la producción agrícola, la obtención de recursos silvestres vegetales o animales, la obtención de materias primas líticas para la manufactura de instrumentos, la cocción y el almacenamiento de alimentos, etc.

Un aspecto importante sobre la actividad pastoril se vincula a su espacialidad. En base a ello, pensamos que posiblemente esta práctica podría haberse vinculado con una arquitectura más bien perecedera, utilizando ramas y espinas para encerrar a los animales y sobre todo para evitar que circulen por los espacios agrícolas a los fines de alimentarse con los cultivos. En algunos sectores de la sierra hemos observado este tipo de corrales, utilizados actualmente para el encierro de cabras. También, en otros casos, se utilizan palos calzados en el suelo con rocas para formar los corrales. Creemos entonces que la construcción de corrales no se habría utilizado la piedra, ya que para algunas construcciones, la roca era imprescindible, como por ejemplo, para las terrazas de cultivo, mientras que los corrales podrían haber sido construidos con materiales perecederos sin ningún inconveniente.

\section{CACERÍA}

En El Taco 19, la relación entre humanos y animales, no sólo se centró en los camélidos domésticos, sino que también cumplieron cierto rol en la reproducción social los animales silvestres, obtenidos principalmente a través de la cacería. En relación a esta práctica, entendemos que en la misma participan varios factores, desde el número de cazadores, las características del paisaje donde se realiza, la etología de las presas, las armas con las que cuentan los cazadores y otros factores, como pueden ser sueños premonitorios, fechas relevantes, etc. (Aschero \& Martínez, 2001; Ratto, 2003; Moreno, 2010, 2013). Claramente, hay aspectos centrales que tienen que ver con el tamaño de las presas y su etología, porque estos factores determinarán gran parte de la manera en que se materializa esta práctica. Para ponerlo en términos simples, no es lo mismo cazar una pequeña ave que un chancho del monte, de igual manera que no es lo mismo contar con armas de fuego que con lanzas arrojadizas e incluso varía enormemente el paisaje donde se realiza esta práctica. Es por ello, que uno de los aspectos principales de la cacería se vincula con los conocimientos, es decir, aquellos saberes que poseen las personas sobre cómo se comportan los animales y más específicamente, cómo reaccionan ante el peligro.

Otro aspecto central de la cacería es la cantidad de cazadores participantes, ya que no es igual ni la estrategia ni las presas pretendidas entre una cacería individual y una colectiva, así como también la forma en que los animales obtenidos son consumidos. Esta situación también definiría posiblemente la frecuencia de las partidas de caza y la organización estaría mayormente mediada por arreglos sociales.

Finalmente, un aspecto principal para considerar es el espacio en el que se desarrollan las cacerías. En el caso de la Sierra de El Alto-Ancasti, este aspecto juega un rol primordial. Pero antes de involucrarnos en este aspecto, es necesario avanzar en la caracterización de algunas de las especies obtenidos por las poblaciones de ET19 a través de esta práctica.

Como comentamos más arriba, contamos con información, todavía escueta, pero que marca una relativa importancia de la obtención de animales silvestres en el conjunto faunístico de ET19. En este sentido identificamos la presencia de cérvidos y de algunos especímenes que no se corresponden a camélidos, pero que serían de animales de mediano y/o gran porte. Considerando el área y su fauna, podrían ser chanchos del monte (Pecari tajacu), cérvidos, puma (Puma concolor), tapires (Tapirus terrestres), etc. Sin embargo, hasta el momento, no hemos podido avanzar en la identificación taxonómica precisa de estos elementos. Además, dentro de esta categoría es posible incorporar los restos de aves.

En el paisaje de la sierra de El Alto-Ancasti, las diferencias ambientales y sus recursos se encuentran marcados por una ladera muy abrupta hacia el oeste, un sector de pastizal de altura, en las estribaciones más altas de la sierra y luego una ladera este, que desciende, con pisos boscosos y de monte serrano hasta llegar a la llanura chaco-santiagueña. Esta situación implica un espacio en el cual las elecciones vinculadas a la materialización de prácticas cinegéticas estarían fuertemente cruzadas por esta característica. En este sentido, en el área de El Taco, al sector vinculado con especies silvestres y cierta recolección de vegetales, se encuentra en espacios con mayor vegetación ubicado en una cota de altura más baja que el espacio de pastizal de altura y que se encuentra dominado 
por un paisaje de monte, donde la dinámica de la vida y la experiencia se modifican completamente de lo que es la reproducción social en el espacio cumbral. Estas zonas no se encuentran separadas por grandes distancias, sino que se trata de espacio ambientales muy diferentes; el primero, marcado por la cotidianeidad de la reproducción social y la transformación del paisaje a partir de las prácticas sociales, mientras que en el segundo se trata de espacios muy poco transformados, con la presencia de monte serrano y bosques de cebil, donde las relaciones entre humanos y animales habrían implicado otras experiencias.

En este marco, las experiencias sociales, así como las relaciones entre humanos y animales habrían sido completamente diferentes a aquellas entabladas en la zona del pastizal de altura. Pensamos que habrían sido travesías poco frecuentes, llevadas adelante en ciertos momentos del año, quizás en conjunto con la obtención de otros recursos silvestres y vinculados posiblemente a la realización de otro tipo de prácticas como, por ejemplo, a la recolección de cebil y a actividades realizadas en espacios rupestres (Quesada \& Gheco, 2011; Gheco, 2012, 2017).

Nos resta conocer muchos aspectos acerca de las estrategias y la tecnología de caza implicadas en este territorio. En relación a la tecnología, contamos con escasa información obtenida de puntas de proyectil, manufacturadas en cuarzo, de diseño triangular, pequeño, algunas con pedúnculo (Moreno \& Egea, 2016), recuperadas en excavación en la localidad arqueológica de Oyola, ubicada en una cota altitudinal más baja, y en un piso boscoso. En el caso de ET19, en el conjunto lítico analizado no se recuperó ninguna de estas piezas (Moreno, 2014). El diseño y tamaño de este tipo de instrumento, se vincularía con el uso de arco y flecha. En el caso de las estrategias de caza, el conocimiento todavía es muy escueto, ya que conocemos poco acerca de la etología de las presas y posibles maneras de cazarlos, pero será un camino que comenzaremos a desandar para conocer y comprender en profundidad esta temática a escala local. Aquí pretendimos llamar la atención sobre la presencia de esta práctica y su articulación en la vida cotidiana de las poblaciones locales.

Finalmente, un espacio particular lo ocupan los roedores recuperados en una cantidad relevante en ET19. La cantidad de especímenes correspondientes a roedores que alcanza el $11,8 \%$ del conjunto total de especímenes óseos, nos llamó la atención considerando la relativa baja cantidad de huesos con marcas de roedor, considerando el estado de conservación del conjunto. Además fue muy baja la identificación de galerías y otras evidencias de la presencia de roedores durante la excavación de ET19. Por ello, proponemos, a modo de hipótesis, el posible ingreso de algunos roedores como parte de la dieta. Este aporte de los roedores ha sido identificado en algunas investigaciones (Santiago, 2004; Escosteguy, 2007; Santini, 2011; Medina et al., 2012). Sin embargo, queda mucho por desarrollar para poder profundizar este aspecto. En primera instancia es necesario avanzar en la caracterización taxonómica de la especie o especies de roedores presentes en el conjunto. Hasta el momento fue posible identificar algunos especímenes asignados a Ctenomys, pero debemos profundizar este análisis para evaluar las potencialidades económicas de los roedores presentes, así como también la presencia de modificaciones culturales en algún ejemplar.

\section{CAMPESINOS, ANIMALES, TERRITORIO, REPRODUCCIÓN}

A lo largo de este trabajo nos hemos centrado en reflexionar sobre las potenciales relaciones entabladas entre animales y humanos en la cumbre de la Sierra de El Alto-Ancasti, durante el primer milenio de la era. Intentamos para ello romper con una visión de las poblaciones locales que pretendía asumir una muy baja densidad poblacional, una fuerte estacionalidad y una única actividad productiva, como era el pastoreo. Las evidencias de diversas líneas de trabajo, muestran una realidad mucho más compleja, donde las poblaciones locales se habrían asentado con una intención de permanecer en el tiempo, con una arquitectura que fija estos sentidos en el territorio y con una fuerte relación con los recursos y el entorno local (Quesada, 2011; Meléndez \& Quesada, 2012; Moreno \& Quesada, 2012; Moreno \& Sentinelli, 2012; Quesada et al., 2012; Zucarelli, 2012; Granizo \& Barot, 2014; Moreno, 2014; Ahumada \& Moreno, 2015-2016; Moreno \& Egea, 2016).

En este marco, entendemos que las relaciones entre humanos y animales fueron mayormente visibles a través del pastoreo de llamas, las que contaban con buenas pasturas distribuidas a lo largo de toda la cumbre y zonas aledañas. El problema radicaba en la interacción de las tropillas con las áreas cultivadas. En este sentido, creemos que la 
preparación de enramadas habría sido una buena opción para lograr evitar el ingreso de las llamas a las terrazas de cultivo.

De igual manera, los rebaños, habrían sido fácilmente protegidos por las poblaciones locales en este ambiente que presenta muchas de las características buscadas por ellos: pastos palatables, agua para beber y protección otorgada por las personas. La cantidad de restos faunísticos identificados en ET19, así como el perfil etario del conjunto nos hace pensar en rebaños conformados por pocos animales, y con un interés dirigido a la obtención de variados recursos. De esta manera, se conformaba una relación cotidiana y de protección entre llamas y pastores, reproducida en el tiempo.

En otro sentido pensamos la relación entre animales silvestres y estas mismas poblaciones, vinculado a otros paisajes, a momentos particulares y a otras prácticas afines a otros recursos e incluso también a otros seres. La cacería se habría centrado en obtener mamíferos de mediano y/o gran porte y su explicación no creemos que debe mirarse desde una posición economicista, vinculada a la resolución de momentos de crisis, sino a prácticas sociales que se vinculan y se desarrollan en este espacio, con estos recursos y con tiempos particulares. El vínculo con las especies silvestres e incluso con este paisaje no domesticado establecería relaciones sociales diferentes a aquellas en las cuales participaban cotidianamente las personas en el sector cumbral de la sierra.

Así vemos que un espacio social, como es la sierra de El Alto-Ancasti, durante el primer milenio de la era, se construye, reproduce, modifica o mejor dicho se experimenta en el vivir y en el entablar relaciones cotidianas con la casa, la chacra, los animales, las plantas, las piedras, el agua y otros seres. Debemos ser capaces de articular y pensar esta vida en términos de múltiples redes de significación y estructuración, donde el pastoreo y la cacería fueron formas de establecer relaciones entre humanos y animales, pero extremadamente significativas para comprender las interacciones sociales cotidianas.

\section{AGRADECIMIENTOS}

Queremos agradecer en primera instancia a todo el equipo de trabajo del proyecto arqueológico de la Sierra de El Alto-Ancasti. Estas investigaciones fueron realizadas gracias al aporte de subsidios de la Secretaría de Ciencia y Técnica de la Universidad Nacional de Catamarca y la Agencia Nacional de Promoción Científica y Tecnológica. Queremos agradecer a Julián Mignino y Andrés Izeta (IDACOR) y a Adan Tauber, Ricardo Torres y a todo el personal del Museo de Paleontología de la Facultad de Ciencias Exactas y Naturales (UNC) por la colaboración en la identificación taxonómica. Finalmente a los editores del volumen por la invitación a participar del mismo.

\section{REFERENCIAS}

Ahumada, M. \& Moreno, E. 2015-2016: La escala doméstica y los animales. Tratamiento diferencial de partes esqueletarias y distribución diferencial intra-sitio en El Taco 19 (El Alto-Ancasti, Catamarca). Anales de Arqueología y Etnología 70/71: 105-118.

Arhem, K. 2001: La red cósmica de la alimentación. La interconexión de humanos y naturaleza en el Noroeste de la Amazonía. En: Descola, P. \& Palssón, G. (eds.): Naturaleza y Sociedad. Perspectivas antropológicas: 80-100. Editorial Siglo Veintiuno, México.

ARnold, D. \& Yapita, J. 1998: Río de vellón, río de canto. Cantar a los animales, una poética andina de la creación. ILCA/Hisbol, La Paz.

Aschero, C. \& Martinez, J. 2001: Técnicas de caza en Antofagasta de la Sierra, Puna meridional, Argentina. Relaciones de la Sociedad Argentina de Antropología 26: 215-241.

BAROT, C. 2017: Las vasijas en la vida diaria. Análisis morfológico funcional del material cerámico de una casa emplazada en las Sierras de El Alto-Ancasti (Siglos VII y VIII d.C.). Tesis de licenciatura inédita. Escuela de Arqueología, Universidad Nacional de Catamarca.

Behrensmeyer, A. 1978: Taphonomic and ecologic information from bone weathering. Paleobiology 1(2): 150-162.

Bettinger, R. 1991: Hunter-gatherers. Archaeological and Evolutionary Theory. Plenum Press, New York.

BinFORD, L.R. 1980: Willow smoke and dog's tails: hunter-gatherers settlement systems and archaeological site formation. American Antiquity 45: 4-20.

- 1981: Bones, Ancient Men and Modern Myths. Academic Press, New York.

Bugallo, L. \& Tomasi, J. 2012: Crianzas mutuas. El trato a los animales desde las concepciones de los pastores 
puneños (Jujuy, Argentina). Revista Española de Antropología Americana 42(1): 205-224.

Chaix, L. \& Méniel, P. 2005: Manual de Arqueozoología. Editorial Ariel, Barcelona.

Dantas, M. 2010: Arqueología de los animales y procesos de diferenciación social en el Valle de Ambato, Catamarca, Argentina. Tesis doctoral inédita. Facultad de Filosofía y Humanidades. Universidad Nacional de Córdoba.

Descola, P. 2004: Las lanzas del crepúsculo. Relatos jíbaros. Alta Amazonía. Fondo Cultura Económica, Buenos Aires.

Dlugosz, J.C. 2005: Prospecciones arqueológicas en los sitios Los Pedraza y Los Corpitos, Dpto. El Alto, Pcia. de Catamarca. Trabajo Final de la Carrera de Arqueología. Facultad de Ciencias Naturales e Instituto Miguel Lillo. Universidad Nacional de Tucumán. San Miguel de Tucumán.

Escosteguy, P. 2007: Los roedores en la localidad arqueológica La Guillerma y los sitios San Ramón 7 y Río Luján. La Zaranda de Ideas 7: 21-39.

GHECO, L. 2012: Una historia en la pared. Hacia una visión diacrónica del arte rupestre de Oyola. Tesis de Licenciatura inédita. Escuela de Arqueología, Universidad Nacional de Catamarca.

- 2017: El laberinto de las paredes pintadas. Una historia de los abrigos con arte rupestre de Oyola, Catamarca. Tesis doctoral inédita. Facultad de Filosofía y Humanidades, Universidad Nacional de Córdoba.

Gordillo, I.; Calomino, E. \& Zuccarelli, V. 2010: En el cercano oriente: el borde como centro. Arqueología en el dto. El Alto, Catamarca. Trabajo presentado en el XVII Congreso Nacional de Arqueología argentina. Mendoza.

Gordillo, I. \& ZucCARELli, V. 2013: Estructuración del paisaje en las tierras altas septentrionales. Trabajo presentado al I Taller de Arqueología de Ancasti y Áreas Aledañas. Tapso, 3 al 5 de diciembre de 2013.

Granizo, M.G. \& Barot, C. 2014: Cambiando Escalas. De cerámicas regionales a locales y domésticas en las serranías de El Alto-Ancasti. Trabajo presentado en las Primeras Jornadas Regionales y Terceras Internas de Antropología. Facultad de Humanidades, Universidad Nacional de Salta. Salta.

HABER, A. 2006: Una arqueología de los paisajes puneños. Domesticidad, interacción e identidad en Antofalla. Primer y segundo milenios d.C. Jorge Sarmiento Editor. Universitas Libros, Córdoba.

- 2007: Arqueología de uywaña. Un ensayo rizomático. En: Nielsen, A.; Rivolta, C.; Seldes, V.; Vázquez, M. \& Mercolli, P. (eds.): Producción y circulación prehispánicas de bienes en el Sur Andino: 13-36. Editorial Brujas, Córdoba.
INGOLD, T. 1987: The appropiation of nature. Essays of human ecology and social relations. University of Iowa Press, Iowa City.

- 1988: Introduction. In: Ingold, T. (ed.): What is an Animal?: 1-16. Unwin Hyman, London.

Izeta, A.; Otaola, C. \& Gasco, A. 2009: Estándares métricos y variabilidad en falanges proximales de camélidos sudamericanos. Su importancia como conjunto comparativo para interpretaciones en arqueología. Revista del Museo de Antropología 2: 169-180.

KRISCAUTZKY, N. 1996-1997: Sistemas productivos y estructuras arqueológicas relacionadas con la producción agropecuaria en el valle de Catamarca. Shincal 6: 65-69.

Lyman, L. 1994: Vertebrate Taphonomy. Cambridge manuals in Archaeology. Cambridge University Press, Cambridge.

MarciniaK, A. 1999: Faunal Materials and Interpretive Archaeology Epistemology Reconsidered. Journal of Archaeological Method and Theory 6(4): 293-320.

Medina, M.; Teta, P. \& Rivero, D. 2012: Burning damage and small-mammal human consumption in Quebrada del Real 1 (Córdoba, Argentina): an experimental approach. Journal of Archaeological Science 39(3): 737-743.

Meléndez, S. \& Quesada, M. 2012: Estrategias Empresariales y Tácticas Campesinas en Mina Dal. Vestigios-Revista Latino-Americana de Arqueologia Histórica 6(1): 157-186.

Mengoni Goñalons, G. 1988: Análisis de materiales faunísticos de sitios arqueológicos. Xama 1: 71-120.

- 1991: La llama y sus productos primarios. Arqueología 1: 179-196.

- 1999: Cazadores de guanacos de la estepa patagónica. Sociedad Argentina de Antropología, Buenos Aires.

Moreno, E. 2010: Arqueología de la caza de vicuñas en el área del Salar de Antofalla, Puna de Atacama. Una aproximación desde la arqueología del paisaje. Tesis doctoral inédita. Facultad de Ciencias Naturales y Museo. Universidad Nacional de La Plata.

- 2013: Estrategias de caza y paisajes culturales en Antofagasta de la Sierra, Catamarca. Comechingonia 17(2): 95-121.

- 2014: Materias primas, instrumentos líticos y prácticas domésticas en las serranías de El Alto-Ancasti, Catamarca. Cuadernos del Instituto Nacional de Antropología y Pensamiento Latinoamericano, Serie Especial N² vol 2: 141-160.

Moreno, E. \& EgEA, D. 2016: Visitas en el tiempo. Tecnología lítica de una cueva con arte rupestre en el este catamarqueño. Arqueología 22(1): 223-232. 
Moreno, E. \& QuesadA, M. 2012: Análisis Preliminar del Conjunto Arqueofaunístico de El Taco 19. Sierras de El Alto-Ancasti. Comechingonia. Revista de Arqueología 16(2): 155-162.

Moreno, E. \& Sentinelli, N. 2012: Tecnología Lítica en las Sierras de El Alto-Ancasti, Catamarca. Cuadernos de la Facultad de Humanidades y Ciencias Sociales No45: 95-105.

NAZAR, D. 2003: Relevamiento arqueológico de la zona austral de la sierra de Ancasti (Provincia de Catamarca). CENEDIT, Universidad Nacional de Catamarca.

NiElsen, A. 1996: Competencia territorial y riqueza pastoril en una comunidad del sur de los Andes Centrales (Depto. Potosí, Bolivia). Zooarqueología de camélidos 2: 53-74.

- 2009: Pastoralism and the non-pastoral world in the late pre-columbian history of the southern Andes (1000-1535). Nomadic People 13(2): 17-35.

Pérez Gollán, J.A. 1994: El proceso de integración en el valle de Ambato: Complejidad Social y Sistemas Simbólicos. Rumitacana 1: 33-44.

Quesada, M. 2011: La periferia desde la periferia. Paisajes Campesinos en las serranías de El Alto Ancasti ( $1^{\circ}$ milenio d.C.). Trabajo presentado al III Taller Internacional de Arqueología del NOA y Andes Centro Sur. Arqueología y Etnohistoria de la Vertiente Oriental de los Andes de Argentina, Bolivia y Perú. Facultad de Humanidades y Ciencias Sociales, UNJu., CONICET, ANPCyT. San Salvador de Jujuy.

Quesada, M. \& Gastaldi, M. 2013: Devenir Casa. Trabajo presentado en el VII Congreso de la Asociación de Estudios Bolivianos.

QuesadA, M. \& GHECo, L. 2011: Modalidades Espaciales y Formas Rituales. Los Paisajes Rupestres de El Alto-Ancasti. Comechingonia. Revista de Arqueología 15: 17-37.

Quesada, M. \& Zuccarelli, V. 2016: Espacio, tiempo y trabajo. Temporalidad del trabajo agrícola de la Sierra de El Alto-Ancasti, segunda mitad del primer milenio d.C. Trabajo presentado en el XIX Congreso Nacional de Arqueología Argentina. Universidad Nacional de Tucumán.

Quesada, M.; Gastaldi, M.; Granizo, M.; Meléndez, S. \& Revuelta, C. 2008: La ocupación humana en las tierras altas de Ancasti durante el Período de Integración Regional. Trabajo presentado en las IX Jornadas Regionales de Investigación en humanidades y Ciencias Sociales, San Salvador de Jujuy.

Quesada, M.; Gastaldi, M. \& Granizo, G. 2012: Construcción de periferias y producción de lo local en las cumbres de El Alto-Ancasti. Relaciones de la Sociedad Argentina de Antropología XXXVII(2): 435-456.
RatTo, N. 2003: Estrategias de caza y propiedades del registro arqueológico en la Puna de Chaschuil (Departamento de Tinogasta, Catamarca, Argentina). Tesis doctoral inédita. Facultad de Filosofía y Letras, Universidad de Buenos Aires.

Reitz, E. \& Wing, E. 2008: Zooarchaeology. Cambridge University Press, Cambridge.

SANTIAGO, F. 2004: Los roedores en el menú de los habitantes de Cerro Aguará (provincia de Santa Fe): su análisis arqueofaunístico. Intersecciones en Antropología 5: 3-18.

SANTINI, M. 2011: Aprovechamiento de Myocastor coypus (Rodentia, Caviomorpha) en sitios del Chaco Húmedo argentino durante el Holoceno tardío. Intersecciones en Antropología 12: 195-205.

TABOADA, C. 2011: Cultura material, espacialidad y procesos sociales tardíos en la arqueología de Santiago del Estero. Desarrollos locales e interacción. Trabajo presentado en el III Taller de Arqueología y Etnohistoria del NOA y Andes centro sur (TANOA III). San Salvador de Jujuy.

Tomasi, J. 2011: Geografías del pastoreo. Territorios, movilidades y espacio doméstico en Susques (provincia de Jujuy). Tesis de doctorado en Geografía, Facultad de Filosofía y Letras, Universidad de Buenos Aires.

Vicent García, J. 1991: El neolítico. Transformaciones sociales y económicas. Boletín de Antropología Americana 24: 31-61.

Viveiros de CAstro, E. 1996: Os pronomes cosmológicos e o perspectivismo amerindio. Mana 2(2): 115144.

- 2004: Perspectivismo y multinaturalismo en la América Indígena. En: Surrallés, A. \& García Hierro, P. (eds.): Tierra Adentro. Territorio Indígena y Percepción del entorno: 37-80. Tarea Gráfica Educativa, Lima.

WheEler, J. 1999: Patrones prehistóricos de utilización de camélidos sudamericanos. Boletín de Arqueología PUCP (3): 297-305.

WING, E. 1972: Utilization of Animal Resources in the Peruvian Andes. In: Izumi, S. \& Terada, K. (eds.): Andes 4. Excavations at Kotosh, Peru 1963 and 1966: 327-354. University of Tokyo Press, Tokio.

Yacobaccio, H.; Madero, C.; Malmierca, M. \& ReiGADAS, M. 1997-98: Caza, domesticación y pastoreo de camélidos en la Puna Argentina. Relaciones de la Sociedad Argentina de Antropología XXII-XXIII: 389-418.

ZucCARelli, V. 2012: Paisajes de producción y reproducción en el Dpto. El Alto-Ancasti, Catamarca, durante el Período de Integración Regional (ca. 600-1100/1200 D.C): usos del GIS en la Arqueología de los paisajes agrarios. Tesis de Licenciatura inédita. Facultad de Filosofía y Letras, Universidad de Buenos Aires. 